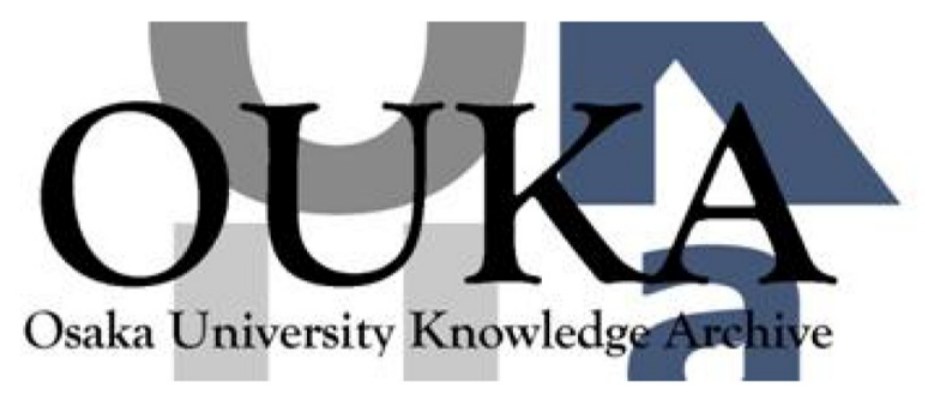

\begin{tabular}{|c|l|}
\hline Title & $\begin{array}{l}\text { Pressure sensor based on freely suspended } \\
\text { ferroelectric liquid crystal fi lm }\end{array}$ \\
\hline Author(s) & $\begin{array}{l}\text { Yablonskii, S. V.; Nakano, K.; Mikhailov, A. S. } \\
\text { et al. }\end{array}$ \\
\hline Citation & Applied Physics Letters. 80(4) p.571-p. 573 \\
\hline Issue Date & $2002-01-25$ \\
\hline oaire:version & VoR \\
\hline URL & https://hdl. handle.net/11094/75834 \\
\hline rights & \\
\hline Note & \\
\hline
\end{tabular}

Osaka University Knowledge Archive : OUKA

https://ir. Library. osaka-u. ac. jp/

0saka University 


\section{Pressure sensor based on freely suspended ferroelectric liquid crystal film}

Cite as: Appl. Phys. Lett. 80, 571 (2002); https://doi.org/10.1063/1.1430270

Submitted: 06 April 2001 . Accepted: 24 October 2001 . Published Online: 25 January 2002

S. V. Yablonskii, K. Nakano, A. S. Mikhailov, M. Ozaki, and K. Yoshino

\section{ARTICLES YOU MAY BE INTERESTED IN}

Freely suspended liquid crystal film transfer: A new method of creating thin smectic films on solid substrates

Applied Physics Letters 59, 917 (1991); https://doi.org/10.1063/1.106300

A novel boundary layer sensor utilizing domain switching in ferroelectric liquid crystals

Review of Scientific Instruments 62, 474 (1991); https://doi.org/10.1063/1.1142090

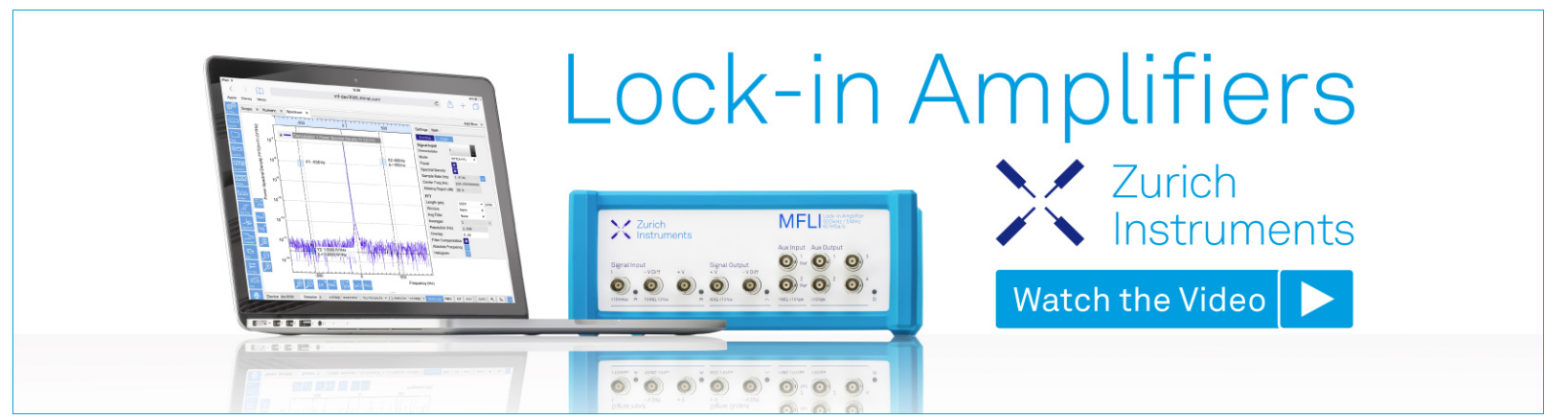




\section{Pressure sensor based on freely suspended ferroelectric liquid crystal film}

S. V. Yablonskii

Department of Electronic Engineering, Graduate School of Engineering, Osaka University, 2-1 Yamada-Oka, Suita, Osaka 565-0871, Japan and Institute of Crystallography, Russian Academy of Science, 117333 Leninskii pr. 59, Moscow, Russia

K. Nakano

Department of Electronic Engineering, Graduate School of Engineering, Osaka University, 2-1 Yamada-Oka, Suita, Osaka 565-0871, Japan

A. S. Mikhailov

Institute of Crystallography, Russian Academy of Science, 117333 Leninskii pr. 59, Moscow, Russia

M. Ozaki ${ }^{\mathrm{a})}$ and K. Yoshino

Department of Electronic Engineering, Graduate School of Engineering, Osaka University, 2-1 Yamada-Oka, Suita, Osaka 565-0871, Japan

(Received 6 April 2001; accepted for publication 24 October 2001)

We report on a pressure gauge based on a vibrating, freely suspended ferroelectric liquid crystal film, which shows some promise of being sensitive to the pressures in the range from 50 to $10^{5} \mathrm{~Pa}$. The gauge indicates changes in resonant frequency of the film caused by changes of air pressure. Pressure measurements using such sensor have been carried out. (C) 2002 American Institute of Physics. [DOI: 10.1063/1.1430270]

Contrary to a liquid crystal sandwiched between rigid substrates, the shape of a freely suspended film can be easily changed by small external forces. Such changes are detected optically using the experimental setup shown in Fig. 1(a). It provides an example of a very sensitive system, which can be used for precise measurements of the mechanical characteristics of the film itself. For instance, by monitoring the resonant frequencies of drumhead vibrations of freely suspended films Miyano succeeded in direct measurements of the surface tension of smectic- $A$ and smectic- $B$ phases. ${ }^{1}$ The films are usually considered as perfect membranes in the Rayleigh sense and their drumhead vibrations obey to 2D wave equation. ${ }^{2-4}$ At the same time, there is some evidence that real freely suspended films are complex objects strongly dependent on their internal structure and boundary conditions. For example, Pieranski et al. pointed out the influence of 3D elasticity of the smectic film on the tune of the membrane. ${ }^{3}$ The nonlinear boundary effects related to meniscus of the liquid crystal freely suspended films have been observed by Brazovskaya et al. ${ }^{5}$ and Cladis et al. ${ }^{6}$ Boudaoud et al. showed that soap films could adapt its mass distribution to the forcing frequency that gives them behavior very different from Rayleigh's membranes. ${ }^{7}$ In addition, freely suspended ferroelectric liquid crystal films manifest very curious phenomenon, so-called electromechanical effect, when alternating electric field is applied along the smectic layers. ${ }^{8-11}$ In this case an interaction of the electric field with ferroelectric liquid crystal film, spanned on the rectangular frame, gives rise to the mechanical vibrations with a discrete spectrum of the pure tones: ${ }^{2}$

$$
\nu=\frac{1}{2} \sqrt{\frac{2 \sigma}{\rho_{s}}\left(\frac{n^{2}}{a^{2}}+\frac{m^{2}}{b^{2}}\right)},
$$

${ }^{a)}$ Electronic mail: ozaki@ele.eng.osaka-u.ac.jp where $a$ and $b$ are, respectively, the length and the width of the film, $\sigma$ is the surface tension, $\rho_{s}=\rho N l$ is the $2 \mathrm{D}$ surface mass density, $N$ stands for the number of the smectic layers, $\rho$ is the bulk density of the liquid crystal, and $l$ is interlayer distance. The integer $n$ and $m$ denote the normal modes.

The sophisticated mechanism ${ }^{12,13}$ for the occurrence of the mechanical vibration in ferroelectric liquid crystals implies the coupling of the azimuth director angle $\varphi_{\mathrm{LC}}$ with the velocity of the center mass of liquid crystal molecules $v$ as shown in Fig. 1(b). The rotation of the director under the action of electric field $E$ gives rise to "backflow," which induces a viscous shear stress $\sigma_{x y}$ disturbing the surface of the film.

In this letter we report on the strong influence of the external air pressure on the electromechanical effect and demonstrate ferroelectric liquid crystal freely suspended film-based pressure sensor.

The freely suspended films were drawn across a plane aperture made in a glass slide of $2 \mathrm{~mm}$ thickness. The process of the preparation of freely suspended films has been described elsewhere. ${ }^{14}$ As the liquid crystal we used commercial ferroelectric liquid crystal mixture CS-1029, ${ }^{15}$ which has the advantage of being in the smectic $C^{*}$ at room temperature. The film thickness was measured by means of reflection spectroscopy. ${ }^{16}$ The reflection spectra were obtained with the aid of multichannel spectrum analyzer. The film holders mounted in the heating stage were contained in a vacuum chamber and the film can be stabilized at a given temperature to $\pm 0.1{ }^{\circ} \mathrm{C}$. The vacuum in the chamber was provided by a mechanical roughing pump and measured with piezoelectric and mercury pressure gauges.

In the experiment the alternating voltage applied across the electrodes excited the vibration modes. The laser beam was reflected from the surface of the film. The angular deviations of the beam were measured with the aid of a position-sensitive detector comprising a slit diaphragm and 


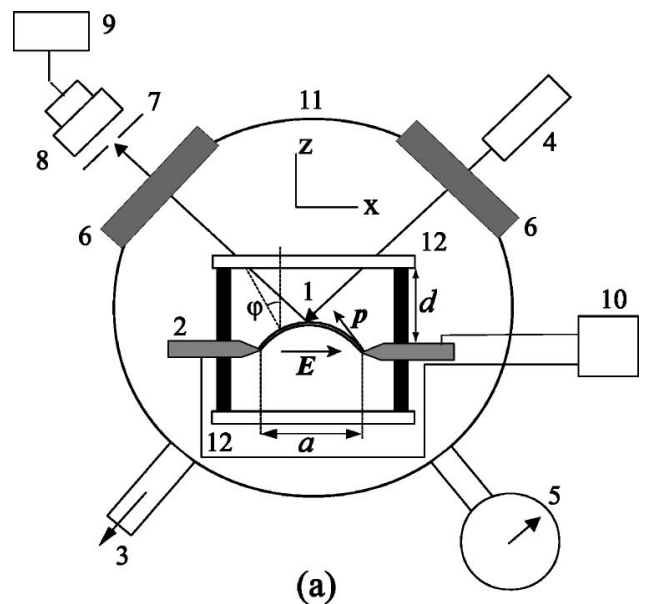

(a)

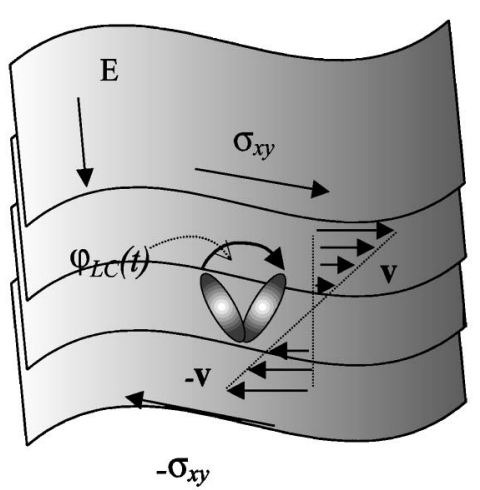

(b)

FIG. 1. (a) Schematic diagram of the experiment: (1) ferroelectric freely suspended liquid crystal films (CS-1029); (2) two Al electrodes; (3) pump; (4) He-Ne laser ( $s$ polarization); (5) manometer; (6) two quartz windows; (7) slit diaphragm; (8) photodiode; (9) lock-in amplifier; (10) audio frequency generator; (11) vacuum chamber, (12) two glasses placed near the film surfaces at the variable distance $d$. (b) Illustration of the coupling between electric field $E$, rotation of director FLC through angle $\varphi_{\mathrm{LC}}(t)$, backflow $v$, and viscous stress $\sigma_{x y}$.

photodiode, as shown in Fig. 1(a). The photodiode response current was analyzed by a Fourier spectrometer and a lockin-amplifier tuned to the first and second harmonics of the sinusoidal voltage applied to the film. The $z$ displacement of the film was sensitive to the polarity of applied electric field; therefore the linear electromechanical effect was basically detected. The amplitude of the second harmonic of the optical response comprised only an insignificant part of the total signal (less than 2\%).

Results of the crucial experiment are shown in Fig. 2. This figure presents the spectra of the linear electromechanical effect for several values of air pressure. When the pressure decreased, the spectra shifted dramatically to the high frequencies. (Correspondingly, the spectrum shifted to the low frequencies, when pressure exceeded normal atmospheric pressure, as shown in Fig. 3. Figure 3 demonstrates both left and right frequency shifts in accordance with positive or negative values of the differential pressure.)

To understand this phenomenon let us analyze probable mechanisms responsible for the frequency shift. First, it is necessary to take into account the mass of ambient gas involved into the motion together with the film. The associated air mass significantly increases the effective $2 \mathrm{D}$ mass density of the film: ${ }^{1} \rho_{s}=\rho_{\mathrm{LC}} h+\rho_{\text {air }} H$, where $h$ and $H$ are the thickness of the film and air loading layer correspondingly. Sec-

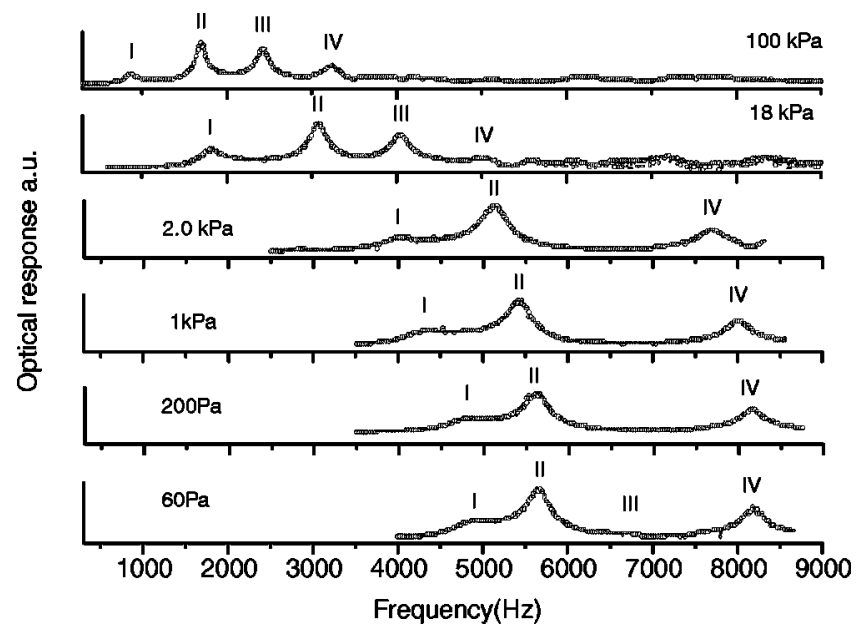

FIG. 2. Spectra of the linear electromechanical effect of a freely suspended liquid crystal film for the different values of air pressure: (rectangular film$2 \times 10 \mathrm{~mm}^{2}, \mathrm{CS}-1029, N=57$ layers, $T=25^{\circ} \mathrm{C}$ ).

ond, to estimate the influence the aerodynamic drag on the motion of the freely suspended films the velocity pressure $\rho_{\text {air }} z_{t}^{2} / 2$ should be compared with inertial term $\rho_{s} z_{t t}^{2}$ (here subscript $t$ denotes the time derivative). For the sinusoidal change of the transverse displacement $z=A_{0} \sin \omega t$ the aerodynamic drag can be essential if $\rho_{\text {air }} z_{t}^{2} / 2$ is comparable with $\rho_{s} z_{t t} \quad$ or $\quad \rho_{\text {air }} \omega^{2} A_{0}^{2} / 2 \sim \rho_{s} h \omega^{2} A_{0}$, that gives $A_{0}$ $\sim 50 \rho_{\mathrm{LC}} h / \rho_{\text {air }}$. Setting $N=50, l=4 \mathrm{~nm}, \rho_{\mathrm{LC}} / \rho_{\text {air }}=10^{3}$ we find $A_{0} \sim 8 \mathrm{~mm}$. In our experimental case oscillation amplitude $A_{0}$ was about $1 \mu \mathrm{m}$, so that velocity pressure can be neglected. Indeed, the linear dependence of the electromechanical effect versus amplitude of electric field (Fig. 4) provides support for the infinitesimal influence of the nonlinear term $\rho_{\text {air }} z_{t}^{2} / 2$ on the motion of the film.

The replacing air in the chamber by helium, possessing a considerably smaller density $\left(\rho=0.178 \mathrm{~g} / \mathrm{cm}^{3}\right)$ than air, shifted the mechanical resonance $f_{1 \max }=1830 \mathrm{~Hz}$ to the high frequency $f_{2 \text { max }}=3140 \mathrm{~Hz}$ in accordance with the decreasing gas medium density (Fig. 5). Knowing two resonant frequencies (for the two different loading masses) allows us to evaluate $H$ and $h$ to be of $0.9 \mathrm{~mm}$ and $0.3 \mu \mathrm{m}$ correspondingly. It is worth noting that the independent experiment with the thick solid reeds $(50 \mu \mathrm{m})$ showed minor loading effect,

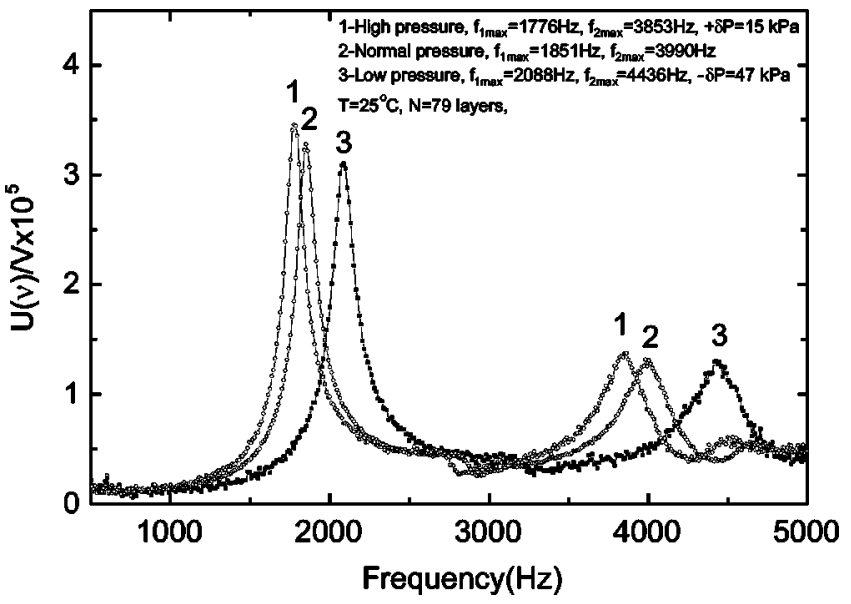

FIG. 3. Spectra of the linear electromechanical effect of a freely suspended liquid crystal film measured for three values of the differential air pressure: $\delta P=+15 \mathrm{kPa}(1), \delta P=0 \quad$ (2), $\delta P=-47 \mathrm{kPa}$ (3). (Circular film $-R$ $=0.15 \mathrm{~mm}, \mathrm{CS}-1029, N=79$ layers, $T=25^{\circ} \mathrm{C}$ ). 


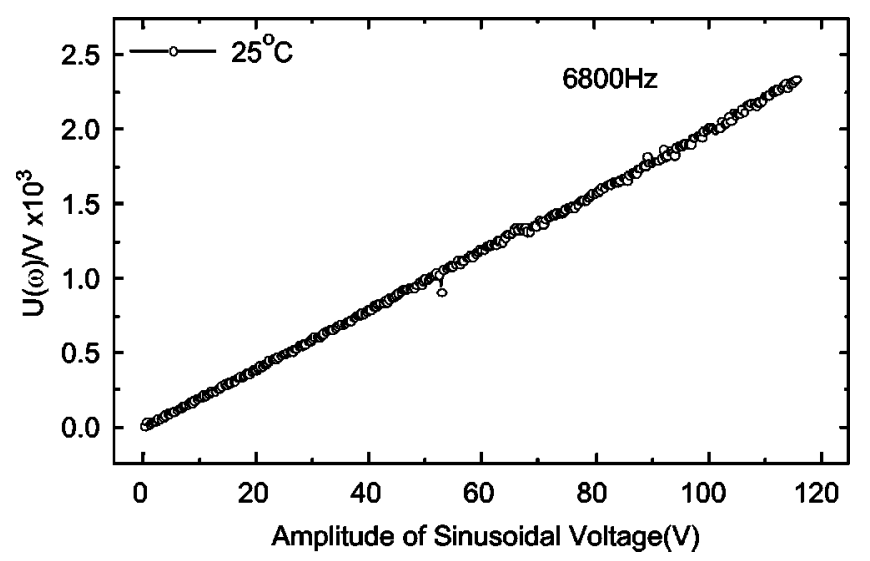

FIG. 4. Linear electromechanical effect vs applied sinusoidal voltage: (rectangular film- $2 \times 10 \mathrm{~mm}^{2}$, CS-1029, $N=48, T=25^{\circ} \mathrm{C}$ ). Measurements were carried out at fixed frequency $\nu=6800 \mathrm{~Hz}$.

amounting to an increase of between one and two percent for the different tones ${ }^{17}$ whereas for the thin (low weight) freely suspended film studied the resonant frequency increased by the factor of 5 , when pressure dropped from $100 \mathrm{kPa}$ to 50 $\mathrm{Pa}$ as shown in Fig. 6. According to formula (1) the frequency shift can be explained by 25 -fold increase of the ratio $\sigma / \rho_{s}$, where $\rho_{s}$ is the strong function of ambient gas pressure.

Thus, the analysis of the spectra of the vibrating ferroelectric freely suspended film as function of absolute pressure leads to conclusion that the liquid crystal ferroelectric film can be successfully used as pressure sensor. The range of measured pressures (800-0.5 Torr) and sensitivity threshold $(0.5$ Torr $)$ of this sensor are almost the same as those of classical $\mathrm{Hg}$ manometer. It should be noted that in our conditions the life expectancy of freely suspended films-based pressure sensor was about several weeks.

It is interesting that the four successive resonant frequencies (shown in Fig. 2) $-\nu_{11}=860 \mathrm{~Hz}, \nu_{22}=1690 \mathrm{~Hz}, \nu_{33}$ $=2420 \mathrm{~Hz}, \nu_{44}=3240 \mathrm{~Hz}$, corresponding to the normal atmospheric pressure were in a good agreement with Eq. (1):

$$
\nu_{11} \approx \nu_{22} / 2 \approx \nu_{33} / 3 \approx \nu_{44} / 4,
$$

where $\nu_{i i}$ denotes the corresponding eigenvalue of the rect-

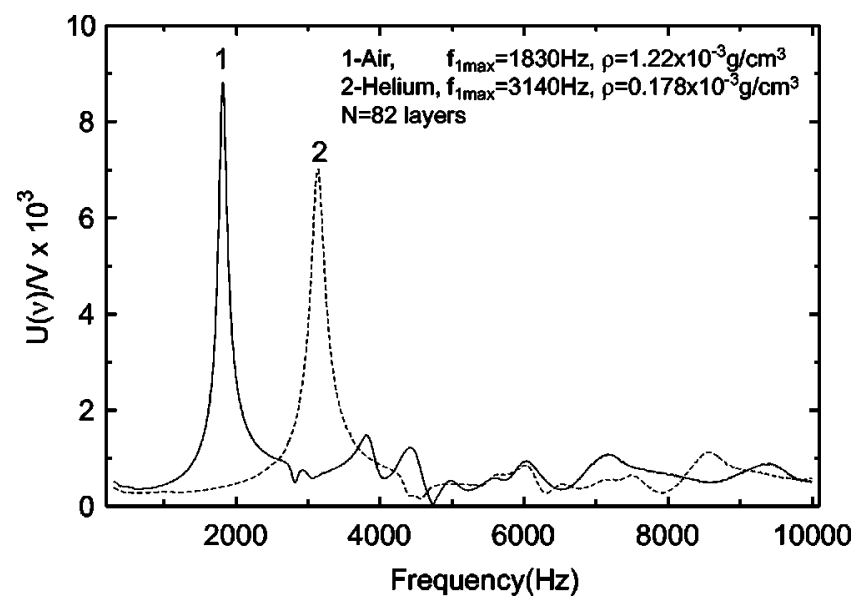

FIG. 5. Spectra of the linear electromechanical effect of a freely suspended liquid crystal film exposed to air (1) and to helium atmosphere (2). In both cases measurements were carried out at normal pressure: (circular film $-R=0.15 \mathrm{~mm}$, CS-1029, $N=82$ layers, $T=25^{\circ} \mathrm{C}$ ).

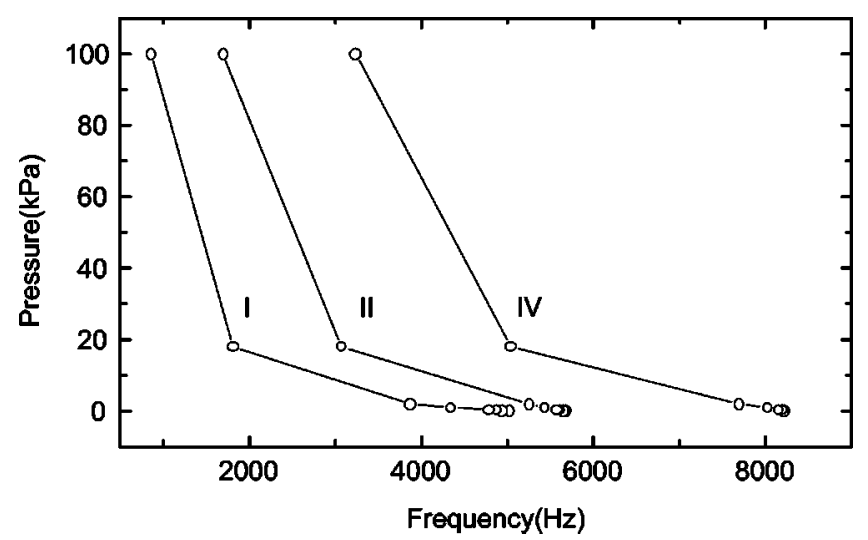

FIG. 6. Air pressure vs positions of the resonant frequencies of a freely suspended film. The numerals near the curves correspond to the numbers of the resonant peaks shown in Fig. 2.

angular film. At the same time, as seen in Fig. 2, a decrease in the pressure was accompanied by a change in the relationship (2). Apparently, the conditions of low pressure (or small loading air mass) increase the role of film inhomogeneities in the region of meniscus. The spectrum of an inhomogeneous linearly elastic film may, in principle, differ from that determined by formula (1).

In conclusion, we have observed the high loading effect in ferroelectric freely suspended liquid crystal films. This effect was manifested by the shift of the resonant frequencies of the films with a decrease in air pressure. The freely suspended film-based pressure gauge operating in the range from $100 \mathrm{kPa}$ to $50 \mathrm{~Pa}$ has been realized. The absence of moving solid parts, simple design and miniature size are essential characteristics of such nanomechanical detector.

This work was supported by a Grant-in-Aid for Scientific Research from the Japan Ministry of Education, Culture, Sports, Science and Technology. S.V.Y. is much indebted to Professor L.M. Blinov for highly stimulating discussions and Dr. S. Palto for the opportunity to use the original software.

${ }^{1}$ K. Miyano, Phys. Rev. A 26, 1820 (1982).

${ }^{2}$ J. W. Rayleigh, The Theory of Sound (MacMillan, London, 1929).

${ }^{3}$ I. Kraus, Ch. Bahr, I. V. Chikina, and P. Pieranski, Phys. Rev. E 58, 610 (1998).

${ }^{4}$ I. Kraus, Ch. Bahr, and P. Pieranski, J. Phys. II 7, 1617 (1997).

${ }^{5}$ M. Brazovskaya, H. Dumoulin, and P. Pieranski, Phys. Rev. Lett. 76, 1655 (1996).

${ }^{6}$ P. E. Cladis, P. L. Finn, and H. R. Brand, Phys. Rev. Lett. 75, 1518 (1995),

${ }^{7}$ A. Boudaoud, Y. Couder, and M. B. Amar, Phys. Rev. Lett. 82, 3847 (1999).

${ }^{8}$ S. V. Yablonskii, T. Oue, H. Nambu, A. S. Mikhailov, M. Ozaki, and K. Yoshino, Appl. Phys. Lett. 75, 64 (1999).

${ }^{9}$ E. I. Demikhov, S. A. Pikin, and E. S. Pikina, Phys. Rev. E 52, 6520 (1995).

${ }^{10}$ S. Uto, E. Tazoh, M. Ozaki, and K. Yoshino, J. Appl. Phys. 82, 2791 (1997).

${ }^{11}$ S. Uto, M. Ozaki, and K. Yoshino, Appl. Phys. Lett. 74, 117 (1999).

${ }^{12}$ A. Jakli, L. Bata, A. Buka, and N. Eber, Ferroelectrics 69, 153 (1986).

${ }^{13}$ A. Jakli and A. Saupe, Liq. Cryst. 9, 519 (1991).

${ }^{14}$ P. Pieranski, L. Beliard, J.-Ph. Tournellec, X. Leoncini, C. Furtlehner, H. Dumoulin, E. Riou, B. Jouvin, J.-P. Fenerol, Ph. Palaric, J. Heuving, B. Cartier, and I. Kraus, Physica A 194, 364 (1993).

${ }^{15}$ The bulk phase sequence for $\mathrm{CS}-1029$ is $\mathrm{Cr}\left(-18^{\circ} \mathrm{C}\right) \mathrm{SmC}^{*}\left(73^{\circ} \mathrm{C}\right) \mathrm{SmA}$ $\left(85^{\circ} \mathrm{C}\right) \mathrm{N}^{*}\left(95^{\circ} \mathrm{C}\right)$ iso.

${ }^{16}$ E. B. Sirota, P. S. Pershan, L. B. Sorensen, and J. Collet, Phys. Rev. E 36, 2890 (1987).

${ }^{17}$ B. S. Berry and W. C. Pritchet, IBM J. Res. Dev. 19, 334 (1978). 